J. theor. Biol. (1976) 57, 419-431

\title{
Towards a Sharper Definition of Energetic Coupling through Integration of Membrane Transport into Bioenergetics $\dagger$
}

\author{
Halvor N. Christensen
}

The Department of Biological Chemistry, The University of Michigan, Ann Arbor, U.S.A.

(Received 31 January 1975, and in revised form 22 May 1975)

Behind the firm discrimination maintained between active and passive transport lies a definition of energetic coupling as a fusion between an exergonic chemical reaction and an uphill transport. In contrast, energetic coupling between paired chemical reactions tends to be defined much more loosely as if the term were merely equivalent to sequential linkage, even though the actual usage may parallel that in transport. This article argues for a sharpening of this definition through integrated consideration of chemi-chemical and chemi-osmotic coupling.

Furthermore, it calls attention to the applicability of energetic coupling to both the backward and forward fluxes of the energized transport. When two parallel but distinct active transport systems act on the same solute, one is likely to operate more steeply uphill than the other. The situation then easily arises, and is probably widespread, whereby entry occurs largely by the first process and exodus by the reversal of the second, still energetically linked. In this way cases of chemi-osmoti-chemical coupling probably arise, beyond the one proposed by Mitchell. Presumably the term retention process has in the past unknowingly (and illogically) referred to the second transport process. The "uncoupling" of an active transport does not tend simply to convert it to a facilitated diffusion, and both fluxes are likely to be modified. Accordingly, measure of only one flux will not describe a change in energy transfer.

\section{Introduction}

Biologists are manifestly encountering some problems in discussing the transfer of energy from one metabolic process to another. The first of these problems is an occasional gross failure to understand how severely circumscribed is the means of that transfer in the general biological context. Weber (1974) has posed a provocative statement to call attention to this

$\uparrow$ This research received support through Grant HD01233 from the National Inștitutes of Health, U.S.P.H.S. 
circumscription: "Every chemical compound generated in metabolism is the result of a reaction which runs toward thermodynamic equilibrium with complete independence of any other reaction occurring at the same time."

His statement can be paraphrased for the field of transport: "Every material flow in the living organism, fully described, runs toward thermodynamic equilibrium with complete independence of any other material flow and the progress of any chemical reaction occurring at the same time."

The consequences of the chemical reaction $A \rightarrow B$ that permit a harvesting of the free energy its progress makes available are in the general biological context its tendency to change the amounts of $A$ and $B$ present at given concentrations, nothing else. If the direction $A \rightarrow B$ is the spontaneous one, then the tendency for $A$ to decrease and for $B$ to increase is the only effect that can do work in that context. And only if these changes can somehow cause the progress of what we may formally consider to be another process, $Q \rightarrow R$, thereby producing some other concentration changes, can we in general harvest any of the energy made available by the reaction $A \rightarrow B$.

I suppose that with its indifference to mechanism, thermodynamics (and particularly the thermodynamics of irreversible processes) has seemed to justify a relaxation of the inevitable necessity that energy transfer have a mechanistic basis.

A more subtle difficulty arises in the definition of coupling. What is the relation that must be established between $A \rightarrow B$ and $Q \rightarrow R$, so that this effect can occur? Textbooks of biochemistry customarily say that two reactions are coupled when a product of one of them is a reactant in the other, as in the sequence:

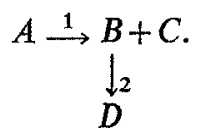

Here the spontaneous progress of reaction 1 can raise the concentration of $B$ enough to cause reaction 2 to proceed. Or the spontaneous progress of reaction 2 can lower the concentration of $B$ enough to cause reaction 1 to become spontaneous. We say that the first reaction "pushes" the second, or that the second reaction "pulls" the first. I want to point out that the mere existence of this relation between two reactions does not satisfactorily exemplify what we may best understand as energetic coupling.

\section{Use of Membrane Transport to Analyze Energetic Coupling}

Chemical reactions and the migrations of substances across membranes are virtually identical in the way that they yield free energy, and in the way that this free energy can be harvested or conserved. It is logical therefore 
to consider what transport can teach us about the nature of energetic coupling. The equations for the free energy changes in the two cases differ only as to the positions of their equilibria. Let us therefore substitute for one of the steps in our reaction sequence above, a passive migration across a membrane, thus:

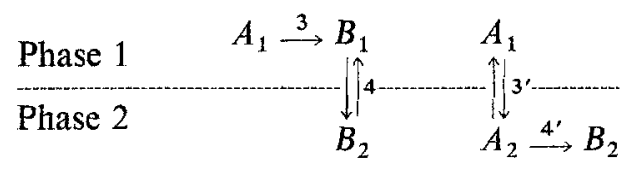

One of the reactants in the reaction $A \rightarrow B$ is able here to penetrate the membrane and to pass freely from one phase to the other. In these cases, the linkage sketched can cause more $B$ to form than would otherwise be generated. If the reaction is initially spontaneous, it can "push" the transport in one case, and "pull" it, in the other. Conversely, if the transport is spontaneous, it can either "pull" (left) or "push" the chemical reaction.

Transport researchers refuse, however, to identify this behavior as active transport, and they would object if you were to claim that the chemical reaction is energetically coupled with the transport. We make a sharp distinction between the situation pictured here and active transport. We insist that the transported solute must be made to migrate against its concentration gradient, or more precisely, against the gradient of its electrochemical potential. Transport workers say that all that exists between steps 1 and 2 in the preceding schemes is sequential linkage and not energetic coupling. Should we not fecl this same distinction for the sequential linkage of reactions 1 and 2 ?

The type of coupling between two reactions that would be parallel to active transport can be illustrated by a ligase reaction:

$$
\begin{gathered}
\mathrm{ATP} \stackrel{\mathrm{H}_{2} \mathrm{O}}{\longrightarrow} \mathrm{AMP}+P P_{i}, \quad \Delta G^{\circ}=-8000 \mathrm{cal} / \mathrm{mol} \\
M+N \rightarrow M N, \quad \Delta G^{\circ}=+3500 \mathrm{cal} / \mathrm{mol} .
\end{gathered}
$$

At the real concentrations of $M$ and $N$ the latter reaction is unable to produce a usable concentration of $M N$. The situation is of course not improved by carrying out the reaction in the presence of ATP simultaneously undergoing hydrolysis. But if we have present an enzyme system that channels the reaction as follows,

$$
\begin{array}{ll}
\mathrm{ATP}+M \rightarrow \mathrm{AMP}-M+P P_{i}, & \Delta G=-2000 \mathrm{cal} / \mathrm{mol} \\
\mathrm{ATP}-M+N \rightarrow \mathrm{AMP}+M N, & \Delta G=-2000 \mathrm{cal} / \mathrm{mol}
\end{array}
$$

we obtain a total reaction, $\mathrm{ATP}+M+N \rightarrow \mathrm{AMP}+P P_{i}+M N$, which is 
also the sum of reactions (5) and (6). Now, however; we include no impossible, endergonic step. A formally endergonic effect has been obtained, however, by energetic coupling. Here we have coupling in more than a platonic sense!

Why does the distinction between sequential linkage and coupling of the kind just illustrated seem so much clearer for specific transport (i.e. for a chemiosmotic coupling, in its broadest sense) than for the relations between two chemical reactions (i.e. for a chemi-chemical coupling)? We intuitively look on the migration of a solute as an event quite different from an associated chemical reaction; hence we easily distinguish a sequential effect of one process on the other, from a direct fusion between the two. In contrast, when we fuse two chemical reactions together, as in (7) and (8), we get another chemical reaction or reaction sequence, and our attention easily shifts from the two reactions we originally perceived, so that now we merely note two new reactions in sequential linkage. The distinction comes then to seem to lie in the eye of the beholder: Does he initially perceive the actual sequence of steps? Then coupling may mean to him merely the behavior arising from the presence of a common intermediating species, as in sequence (3) and (4). Does he instead first perceive two reactions $A \rightarrow B$ and $Q \rightarrow R$, which share no reactant so that sequential linkage is excluded? In that case, coupling to him means the mechanism by which these two reactions are fused together, thus, $A+Q \rightarrow B+R$, which can be exemplified by the sequence (7) and (8)

The essential feature that seems so real for active transport is that through the coupling, a concentration gradient is created, a gradient which shows some tendency to decay spontaneously through leakage of the solute across the membrane. But a conspicuous gradient tends also, so to speak, to be generated by chemi-chemical coupling: levels of $M N$ are formed far higher than we could expect from the concentrations of its precursors, $M$ and $N$, taking into account their affinity for each other. This gradient generally tends also to decay, through a degree of spontaneous breakdown of $M N$ to $M$ and $N$. In addition, both of these energetically coupled processes face the risk of inefficiency from the tendency of the initially perceived exergonic reaction (e.g. reaction 5) to occur in a way such that $M N$ is not formed, or such that the solute is not transported against a gradient. In the presence of an uncoupler, each of these gradients may undergo accelerated decay. In contrast, the concept of the uncoupler has, it seems to me, a dubious relation to sequential linkages. The role of membranes in sequential linkage is usually one of controlling or preventing linkage, whereas for energetic coupling the membrane often plays a role intrinsic to the coupling. 


\section{Further Consideration of Active Transport}

The familiar stipulation that a gradient of a solute across a membrane cannot be generated except by coupling to an exergonic process can be simply illustrated by co-transport (osmotic-osmotic coupling), now studied for over two decades (see Christensen \& Riggs, 1952; Riggs, Walker \& Christensen, 1958; Heinz, 1972), using the accompanying figure. The figure shows a receptor site facing first one side and then the other of the plasma membrane of a cell. This site is filled by the binding of both $\mathrm{Na}^{+}$and a solute $X$, and vacated by the dissociation of both together. Between the binding step and the dissociation step, the site may reorient itself from one surface to the other.

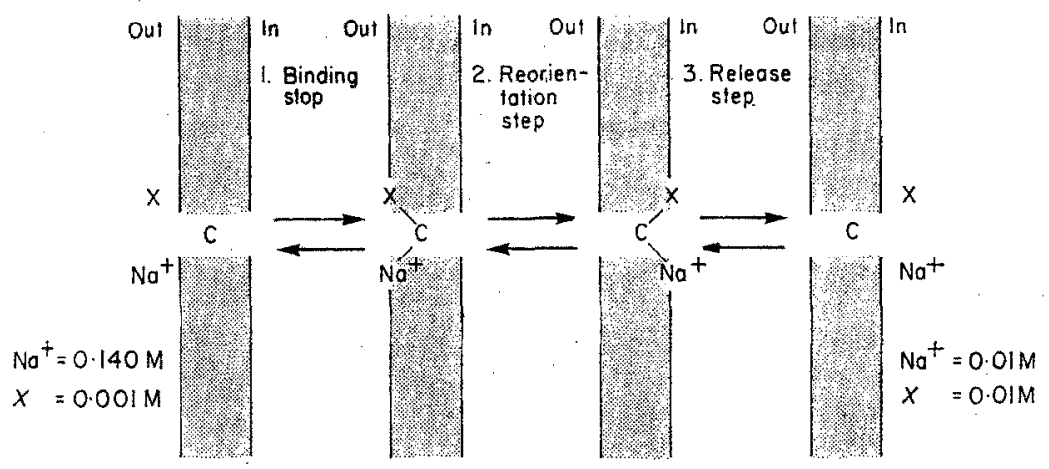

FIG. 1. Osmotic-osmotic coupling: Co-transport between $\mathrm{Na}^{+}$and an organic metabolite, as discussed in the text. How high the gradient of $X$ can rise will depend on whether the coupling is actually stoichiometric, whether there is a transmembrane potential, and whether there is a leak.

If we suppose that a separate process at the same time maintains a $\mathrm{Na}^{+}$ gradient across the membrane, we may see the process,

$$
\mathrm{Na}_{\text {out }}^{+}+X_{\text {out }} \rightarrow \mathrm{Na}_{\text {in }}^{+}+X_{\text {in }}
$$

generating and maintaining a gradient of $X$, in the opposite direction to that of $\mathrm{Na}^{+}$. We may see the process as an active transport of $X$ (as is customary), provided that we focus our attention on the behavior of $X$, although the real process, through a fusion of the down-gradient movement of $\mathrm{Na}^{+}$with the up-gradient movement of $X$, is exergonic or spontaneous. Incidentally, if we were to relax the requirement that the two co-substrates are bound together and released together, we could have a loose and relatively inefficient linkage between the two flows. 
Turning then to the case where a chemical reaction is to drive an active transport (chemi-osmotic coupling), we also need a fusion of these two formally perceived processes,

into one, as follows:

$$
A \rightarrow B ; \quad X_{1} \rightarrow X_{2}
$$

$$
A+X_{1} \rightarrow B+X_{2} \text {. }
$$

The subscripts 1 and 2 mean that $X$ is released into a phase distinct from the one from which it is taken up. This equation does not purport to show the mechanism. We may speculate that this process is carried out by protein oligomers lying within the membrane, taking the behavior of the haemoglobin tetramer as a model for the shifts of sites that can be produced on binding small effector molecules. These oligomers might well bear not only the catalytic sites producing translocation within the oligomeric molecule, but also true enzymatic sites catalyzing the energizing reaction, $A \rightarrow B$. As suggested elsewhere, we might do well to consider the translocating sites as modifier sites to the sites catalyzing the chemical reaction, accepting the principle that the interaction between these two classes of sites must be reciprocal. Given the functional variety and specificity of binding sites on protein molecules, such a process seems to offer no unique difficulties. We do not need, incidentally, to insist that $X$ is translocated all the way across the membrane; an asymmetric translocation across some finite interval within the membrane may prove sufficient.

The stubborn and fruitful discrimination maintained in the use of the term active transport provides a model then for sharply identifying energetic coupling between reactions in its most characteristic aspect.

If the coupled process:

$$
A+Q \rightarrow B+R
$$

takes place in a single-phase system, the energy inherent in the tendency of $A$ to be converted to $B$ would be wasted if $Q$ and $R$ were in fact identical molecules. But if $Q$ and $R$ lie in two different phases, then an effective, active transport can be produced even if they are identical. The enzymologist has often scemcd rcluctant to recognize this simplification obtained on proceeding from the homogeneous to the two-phase system. Deeply impressed by the model of fused exergonic and endergonic reactions in the homogeneous system, he may rather feel that the active transport of a sugar calls for it to appear in phase 2 as a sugar derivative. Energetically, there is no such necessity. If there were, active $\mathrm{Na}^{+}$transport as we know it could not exist.

In the case of the combination of a formally exergonic with a formally endergonic process [say reactions (7) and (8)], the barriers that help maintain 
the efficiency of the fused process are the presence of energies of activation high enough to minimize the spontaneous reaction (3), also spontaneous breakdown of the intermediate AMP $-M$ and the product $M N$. For the osmotic-osmotic linkage,

$$
\mathrm{Na}_{\text {out }}^{+}+X_{\text {out }} \rightarrow \mathrm{Na}_{\text {in }}^{+}+X_{\text {in }}
$$

the resistance of the membrane to any form of passage of $\mathrm{Na}^{+}$or $X$ ("leakage"), except that shown, takes the place of the energy of activation of the more highly spontaneous chemical reactions in the chemical-chemical linkage. In the case of chemiosmotic linkage

$$
A+X_{1} \rightarrow B+X_{2}
$$

the magnitude of both the energies of activation restraining respectively the spontaneous events, $A \rightarrow B$ and $X_{2} \rightarrow X_{1}$, serves to protect the efficiency.

\section{Reversibility of Active Transport}

The third difficulty concerns the understanding of whether transport is reversible and what energy transfer occurs during its reversal. Lehninger's textbook of biochemistry (1970) reads as follows: "Usually active transports are directional and can translocate a solute in but one direction." In discussions of transport, one frequently encounters the intuitively attractive viewpoint inherent in a literal reading of this sentence. The tendency seems to be to expect a second mediated process, or even a simple leak, to serve for exodus. It tempts researchers to try to discriminate between energization through action on the uptake process and through action on a retention process, without full regard as to whether these are separate aspects of a single process, or necessarily distinct processes; also without regard to the question how a process is able to produce retention, and why retention should require energy. It also produces a dubiously significant argument as to whether the steady-state membrane potential arises from an imbalance (a) in the pumping of different cations across the plasma membrane, or (b) in the relaxation fluxes associated with the resultant gradients. We should attribute to active transport the net electrogenic effect of the inherent opposed cation fluxes; their discrimination as to direction seems to have little meaning for this question, as long as we include only the forward and backward fluxes inherent to the active process.

\section{Development}

If the process,

$$
A+X_{1} \rightarrow B+X_{2} \text {, }
$$

is spontaneous in one direction at one set of concentrations of the four 
components, we expect it to be spontaneous in the reverse direction at another set of concentrations:

$$
B+X_{2} \rightarrow A+X_{1} \text {. }
$$

This expectation arises from the real nature of coupling, i.e. the actual fusion of $A \rightarrow B$ to $Q \rightarrow R$ or $X_{1} \rightarrow X_{2}$. Using conventional terms, we may say that the down-gradient flow of $X$ is now driving the conversion of $B$ to $A$. At equilibrium, the process will be spontaneous in neither direction, the two fluxes being equal. The theoretically maximal gradient of $X$ that can be generated at equilibrium is set by the energy available from the tendency of $A$ to be converted to $B$ under the real conditions. If $X$ were now supplied to phase 2 at a concentration exceeding that needed to produce equilibrium, the down-gradient movement of $X$ would cause $B$ to be converted to $A$, a conversion that would be endergonic if it occurred alone. $f$

We should be wrong to suppose that the theoretical gradient attainable is set by a leak or by a separate transport process. If a leak in fact limits the gradient, we can plug the leak and proceed to a still higher gradient. If a separate, outward transport process sets the limit, we can saturate that process with a specific analog, and let the gradient rise still higher. The theoretical maximal gradient cannot, however, be exceeded by sealing a leak, without creating a perpetual machine.

\section{Biological Importance}

This predicted reversibility will set then the maximal gradient that the process can generate, Multicellular organisms regularly show the wide biological importance of the net operation of membrane transports in the downhill as well as in the uphill direction. The liver cell takes up amino acids from its extracellular environment during the post-prandial interval, but during much of the day it releases them again to the blood circulation. All the while, the concentration gradient is strongly in favor of the cytosol, and the same systems seem to mediate the uptake and the release phases. Epithelial cells of the intestine and kidney concentrate amino acids into their interior from both the brush border and the serosal or periplasmic poles, although functionally the net direction of both flows is usually from the brush border to the serosal side. The lesson is clear. We should not expect to read the direction of the net flow from the direction of the gradient.

A related problem reveals that we have been more successful in teaching the organochemical than the physicochemical concepts underlying biochemistry. Transport processes serve to maintain an interval environment

$\dagger$ It is mechanistically possible for the necessary backward path to be in detail not identical with the forward path. 
sufficiently rich in each amino acid to drive the synthesis of proteins and other substances, even in the epithelial cell. Some authors have been tempted to restrict their attention to the essential amino acids even at this subcellular level, confusing the secondary importance on one hand of the ability to synthesize an amino acid, with the overwhelming importance on the other hand of maintaining a sufficient chemical potential of that amino acid to drive the biosynthetic processes. It makes little difference whether the amino acid in question arises from inside or outside the cell under examination. The transport process must in either case maintain an adequate internal concentration of it.

In micro-organisms, the release of cellular amino acids may fall under strong kinetic control through a sequestering of the carrier site (trans inhibition), so that equilibrium is unlikely to be attained. This phenomenon may mask, along with the steepness of the attainable gradients, the reversibility of transport for such organisms.

\section{Steady-states Compared with Equilibria in Active Transport}

The equilibrium of the process,

$$
A+X_{1} \rightarrow B+X_{2}
$$

will not be attained if there is a leak for $X$ through the membrane. Membranes are, however, much less leaky, in the simple sense, than is often supposed, as I have discussed elsewhere (Christensen, 1975). Equilibrium will also be unattainable if $X$ is transported into the cell or organelle by a second process, not so steeply uphill at the ambient concentration:

$$
Q+X_{1} \rightarrow R+X_{2} \text {. }
$$

In this event, it is important to understand that the steady state of the maximal accumulation of $X$ will arise largely from the balance between entry by process (I) and exodus by the reversal of process (II). To seek evidence for that situation we should do well to look for an overall reaction as follows:

$$
A+R \rightarrow B+Q \text {. }
$$

What we encounter here is a generalized form of the Mitchell hypothesis. If a given solute is concentrated across a membrane by two parallel, independent processes, one more strongly concentrative than the other under the real circumstances, then one of the formally defined "driving" reactions will energize the reversal of the other, by way of the transport processes. This deduction makes it highly important to discover whether two parallel active transports are really energized by two independent reactions. If they are, 
then a chemical-osmotic-chemical coupling becomes likely, and one not necessarily restricted to the proton.

Note that energy can be stored by the cell in the form of a gradient, using that term in the broader sense, even if that gradient decays by the same route that formed it. We are familiar with the storage of cellular energy in the form of gradients of the alkali-metal ions, presumably often more tolerable and useful than storage in the form of a hydrogen-ion gradient. For a gradient to represent not only the storage of energy, however, but also the transmission of energy from one reaction to another without the necessity of a change in the level of the gradient, we must have two independent modes of its generation. This feature, apparently not rare, should help identify transports that serve for coupling between chemical reactions.

\section{Will Deletion of the Energy Input for an Active Transport Convert it to Facilitated Diffusion?}

One often sees the energization of transport represented as shown here. A chemical reaction is shown as impinging only on the forward flux of the transport, to make it larger than the backward flux:

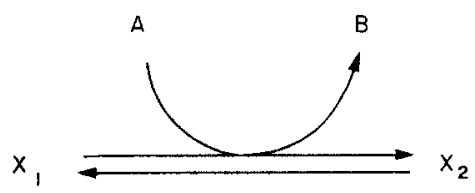

The outward flux is shown as independent of the chemical reaction. This scheme leads to the expectation that an elimination of the chemical reaction would leave us with an unmodified back flux. Since active transport will now be impossible, the forward flux must come to an equal value. The residual transport may be identified as a facilitated diffusion.

The scheme just drawn does not, however, correspond to the equation

$$
A+X_{1} \rightleftharpoons B+X_{2} \text {. }
$$

It sets up no expectation that addition of an excess of $X$ in phase 2 will cause the conversion of $B$ to $A$. Accordingly, the arrow pointing to the left does not represent the thermodynamically essential reversal of the uphill transport. We may instead identify it as a "leak".

The second and third formulations, below, try instead to show the chemical reaction influencing the balance between the two transport fluxes:

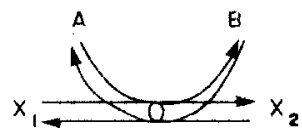

or

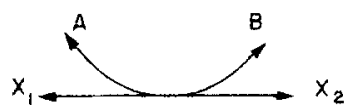


Here the reverse flux is no more independent of the chemical reaction than the forward flux; adding $X$ in excess to phase 2 should cause the conversion of $B$ to $A$; hence, this scheme does correspond to the equation above. But in this case there seems to be no basis for expecting the process to continue as a facilitated diffusion (the forward flux declining until it equals an unchanged backward flux) when the energy input is interrupted. If we remember that energy is transferred to the reaction only in the form of a modified chemical potential of one or more of the reactants, we can understand that prediction to be an oversimplified one. Therefore, a facilitated diffusion uncovered by elimination of the energy input to an active transport could represent an unrelated phenomenon. Cecchini \& Koch (1975) have illustrated the matter nicely in experiments with $E$. coli cells depleted of their readily available energy stores. These cells fail to admit $o$-nitrophenylgalactoside, even by downhill transport, unless an uncoupling agent is added to relieve the proton gradient which is generated otherwise by the associated inward co-transport of $\mathrm{H}^{+}$. Figure 2 shows a scheme proposed by Christensen \& Handlogten

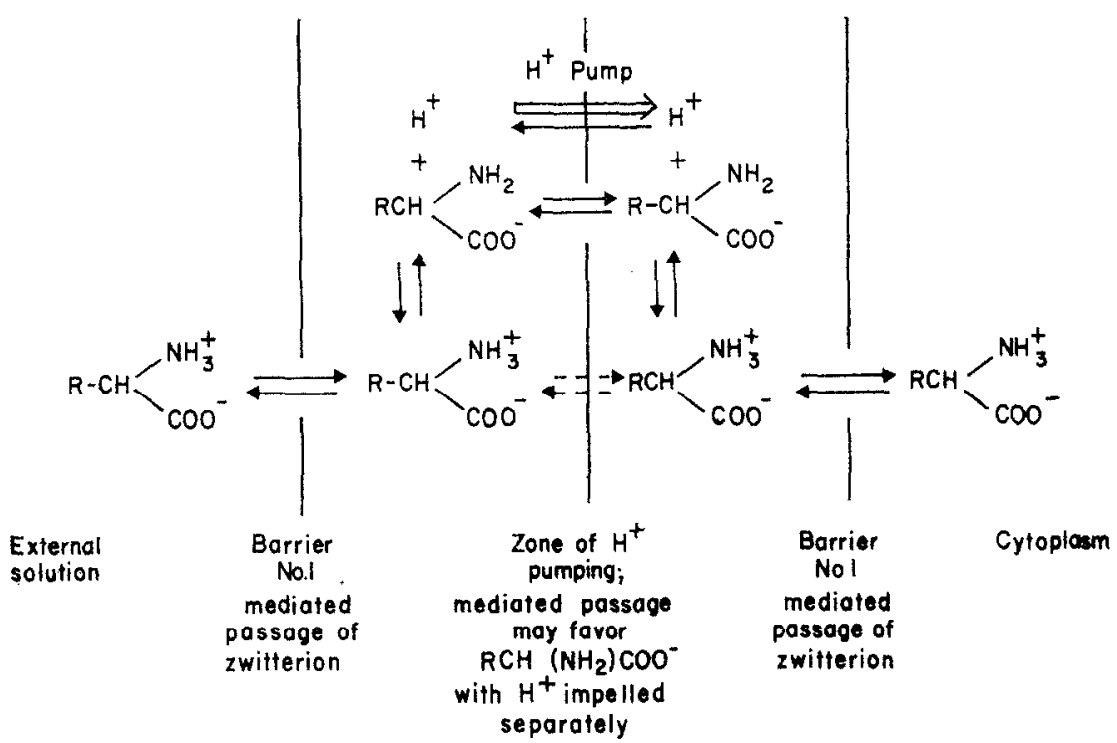

FIG. 2. A hypothesis as to how amino acid transport may generate or exploit an intramembrane gradient of the hydrogen ion. The point made here is that interruption of proton pumping to the right at the top could terminate amino acid transport through a restraint arising from accumulation of $\mathrm{H}^{+}$at the upper right. An uncoupling agent could relieve that restraint. Reproduced with permission from Christensen \& Handlogten (1975). A variant of this hypothesis can make important the charge separation inherent in an intramembrane interconversion between $\mathrm{RCH}\left(\mathrm{NH}_{2}\right) \mathrm{COOH}$ and $\mathrm{RCH}\left(\mathrm{NH}_{3}{ }^{+}\right) \mathrm{COO}^{-}$, e.g., in a pairing to the fundamental charge separation of the proton from the electron. 
(1975) by which a proton gradient within the membrane can be generated or exploited by the movement of amino acids across the membrane. Were proton movements occasioned by net amino acid migration not compensated by another process, e.g. proton pumping, an intra-membrane gradient could soon arise sufficient to check the mediated amino acid movements. A protonophoric uncoupler could relieve this restraint.

Cellular retention processes? The first identification of a so-called retention process to drive transport proved in error. 2,4-dinitrophenol was held to interfere not with the bacterial uptake of galactose, but with its retention (Osborn, McClellan \& Horecker, 1961). Subsequent evidence showed that this substance produced its apparent slowing of galactose exodus by actually inhibiting recapture of the already escaped sugar molecules by a second, unsuspected transport system. Although it is often difficult to be sure what writers mean by a retention process, schemes have been drawn to imply that the energy input to a single transport system can be restricted to the inward, uphill flux, or to the outward, downhill flux. Although one understands that the mutual effect of a change in energy input on the two fluxes may be shifted more to one flux than to the other, for example, through the phenomena of trans stimulation, I wonder whether we can logically expect either flux to be totally unaffected.

Wilson \& Kusch (1972) posed an interesting rhetorical question in discussing mutations in $E$. coli in which the energy input to the $\beta$-galactoside system was presumably eliminated. These mutations led not as expected to a slowed entry but to an accelerated exodus. "If the coupling has no effect on entry, how can the energy coupling provide a useful mechanism for the cell to survive in competition with its neighbors?" The discussion of this question reminds us that it makes little difference for survival whether a steady state gradient is lowered through a slowing of entry or an acceleration of exodus. By the same token, one does not explain how the cystinotic cell accumulates cystine by merely showing an accelerated cystine influx; the other flux must be measured, too, before that matter is explained.

It seems to me possible although not necessary that the mentioned mutations in $E$. coli could have injured the energy input not to the dominant uphill system, but to a less strongly uphill system,

$$
Q+X_{1} \rightarrow R+X_{2} \text {. }
$$

Even in that case it would seem confusing to me to call this second transport system a retention system. At another set of concentrations their roles could conceivably be reversed.

The use of one active transport process by the cell to capture a nutrient and of another active process, working in reverse to allow its restrained release, 
may well permit biological regulation superior to that obtainable with a single reversible process. Systems $A$ and $L$ for ncutral amino acids may well represent this relation. For the epithelial cell, the further opportunity is obtained of distributing the distinct transport entities inequally between the two poles of the cell. What better way to assure that the epithelial cell retains the amino acids it is transporting at sufficient levels for its nutrition than to let that release occur by reversal of a weakly concentrative pump at the basolateral surface?

\section{REFERENCES}

Cecchini, G. \& KoCH, A. L. (1975). J. Bacteriol. 123, 187.

Christensen, H. N. (1975). Biological Transport, Second edn. Pp. 377-380. New York: W. A. Benjamin Co.

Christensen, H. N. \& Handlogten, M. E. (1975). Proc. natn. Acad. Sci. U.S.A. 72, 23. Christensen, H. N. \& Rigas, T. R. (1952). J. biol. Chem. 194, 57.

Heinz, E. (1972). Na-linked Transport of Organic Solutes. Berlin: Springer.

LeHNINGER, A. L. (1970). Biochemistry, p. 605. New York: Worth.

Osborn, M. J., McLellan, JR., W. L. \& HoreCKeR, B. L. (1961). J. biol. Chem. 236, 2585.

Riggs, T. R., Walker, L. M. \& Christensen, H. N. (1958). J. biol. Chem. 233, 1479.

Rotman, B. \& Radojkovic, J. (1964). J. biol. Chem. 239, 3153.

Weber, G. (1974). Ann. N.Y. Acad. Sci. 227, 486.

Wilson, T. H. \& Kusch, M. (1972). Biochim. biophys. Acta 255, 786. 